\title{
Direct assay for endo- $\alpha$-mannosidase substrate preference on correctly folded and misfolded model glycoproteins.
}

\author{
Simone Dedola, ${ }^{a}$ Masayuki Izumi, ${ }^{b}$ Yutaka Makimura, ${ }^{a}$ Akira Seko, ${ }^{a}$ Akiko Kanamori, ${ }^{a}$ \\ Yoichi Takeda, ${ }^{a}$ Yukishige Ito, ${ }^{a, c} *$ and Yasuhiro Kajihara $a, b *$
}

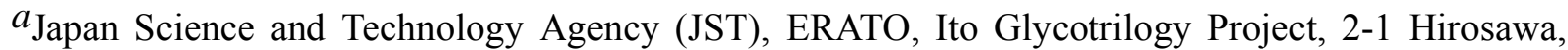
Wako, Saitama, 351-0198 Japan

$b_{\text {Department of Chemistry, Graduate School of Science, Osaka University, 1-1 }}$

Machikaneyama-cho, Toyonaka, Osaka, 560-0043 Japan

${ }^{C}$ Synthetic Cellular Chemistry Laboratory, RIKEN, 2-1 Hirosawa, Wako, Saitama, 351-0198 Japan

\begin{abstract}
We previously reported a unique assay system for UDP-glucose glycoprotein glucosyltransferase (UGGT) toward glycoprotein folding intermediates during the folding process. The assay involved the in vitro folding of both high-mannose type oligosaccharyl crambin, which yielded only the correctly folded glycoprotein form (M9-glycosyl-native-crambin), and its mutant, which yielded misfolded glycoproteins (M9-glycosyl-misfolded-crambin), in the presence of UGGT. The process successfully yielded both mono-glucosylated M9-glycosyl-native-crambin (G1M9-glycosyl-nativecrambin) and M9-glycosyl-misfolded-crambin (G1M9-glycosyl-misfolded-crambin). Here, we report the use of our in vitro folding system to evaluate the substrate preference of Golgi endo- $\alpha$ mannosidase against G1M9-native and -misfolded glycoprotein forms. In our assay Golgi endo- $\alpha$ mannosidase removed Glc- $\alpha-1-3-$ Man unit from G1M9-native and -misfolded-crambins clearly proving that Golgi endo- $\alpha$-mannosidase does not have specific preference for correctly folded or misfolded protein structure.
\end{abstract}




\section{Introduction}

$\mathrm{N}$-Linked oligosaccharides incorporated into proteins can be categorized into three types: high mannose-, complex-, and hybrid-types; the high mannose type oligosaccharides play key roles during the glycoproteins folding process in the Endoplasmic Reticulum (ER) [1]. During glycoprotein biosynthesis in the ER, a high mannose type oligosaccharide bearing three glucose residues is transferred en-bloc from the dolichol phosphate to the nitrogen atom of an asparagine (Asn) side chain belonging to the Asn-Xaa-Ser/Thr consensus sequence (where Xaa can be any amino acid but Pro) of a nascent polypeptide coming from a ribosome (Figure 1) [2].

The newly synthesized glycosylated polypeptides undergo a strict quality control system in which high mannose type oligosaccharides bearing a glucose residue are used as tags for misfolded glycoproteins [1] during their folding process. The quality control system regulating the glycoprotein folding process in the ER consists of a pool of enzymes including calnexin/calreticulin (CNX/CRT), ERp57, glucosidases I and II and UDP-glucose: glycoprotein glucosyltransferase (UGGT), these enzymes accelerate glycoprotein folding and prevent the accumulation of misfolded glycoproteins [3]. CNX/CRT are chaperones in charge of the folding and prevent the aggregation of unstructured polypeptide chains. ERp57 is an oxido-reductase protein dedicated to the formation of disulfide bonds, and glucosidases I and II (GI and GII) provide the de-glucosylation of the first (GI), second and third glucose (GII), respectively. Once glycoproteins are released from their chaperones, they undergo inspection by UGGT, which re-glucosylates misfolded glycoproteins in order to send them back to $\mathrm{CNX} / \mathrm{CRT}$ chaperones for re-folding attempts. Glucosylation/deglucosylation of high mannose type oligosaccharides of glycoproteins seems to be, therefore, a crucial step in the quality control cycle [4], determining which glycoproteins should be sent to one of three following routes: to the Golgi for further glycan-processing, to the ER chaperones for further re-folding attempts or to the ER associated degradation process (ERAD) [1]. 

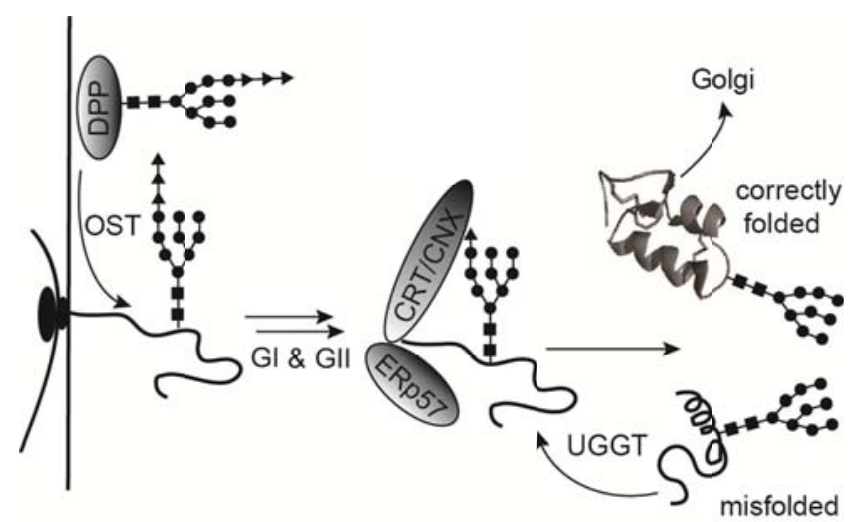

Figure 1. General scheme for glycoprotein quality control cycle in the ER.

In 1987 Spiro et al. discovered an alternative de-glucosylation route $[5,6]$ performed by an endo- $\alpha$-mannosidase located in the cis Golgi. This endo- $\alpha$-mannosidase is capable of removing the Glc- $\alpha-1-3-$ Man disaccharide unit at the non-reducing terminal (A-arm) of high mannose type oligosaccharide (Figure 2). The role of this enzyme has not been completely clarified, but its crystal structure has been recently solved by Davis et al. [4,7].

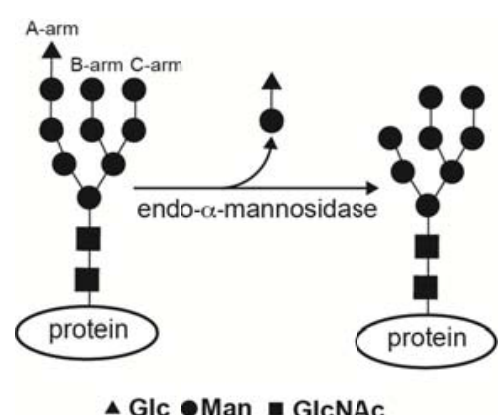

Figure 2. Schematic Golgi endo- $\alpha$-mannosidase activity: cleavages of the last Glc-Man disaccharide from the A-arm of high-mannose type oligosaccharide.

It has also been hypothesized that the endo- $\alpha$-mannosidase could work as a Golgi resident back-up system for the maturation of $N$-glycoproteins that elude the UGGT monitoring step in the ER [8]. In 2006, Torossi et al. demonstrated that endo- $\alpha$-mannosidase could not discriminate between correctly folded or misfolded species of the glycoprotein ( $\alpha 1$-antitrypsin) [9]. However, 
their biological assays were cell based and the misfolded $\alpha 1$-antitrypsin may have been considerably heterogenic in its oligosaccharide structure. While the preparation of G1M9-glycosylnative-glycoproteins and their misfolded forms are generally difficult to obtain either by a cell expression system or by chemical synthesis, we have previously reported a simple protocol to access homogeneous glycoproteins that generates both correctly folded and misfolded G1M9glycoproteins. We therefore decided to exploit such a system to design a conventional assay suitable for the evaluation of endo- $\alpha$-mannosidase substrate specificity.

Our results clearly confirmed that the Golgi endo- $\alpha$-mannosidase cleaves the Glc- $\alpha-1-3-$ Man disaccharide unit from G1M9-highmannose type oligosaccharides of both correctly folded and misfolded glycoproteins without any preference for either one of the substrates.

\section{Results}

\subsection{Design of assays for Golgi endo- $\alpha$-mannosidase}

To elucidate the substrate preference of Golgi endo- $\alpha$-mannosidase we used a mixture of correctly folded and misfolded G1M9-glycoproteins prepared by enzymatic reactions with UGGT. Although it is known that UGGT does not transfer a glucose residue to correctly folded glycoproteins, we fortunately found that, by combining UGGT with our specifically designed unfolded glycopeptides, we were able to achieve a mixture of correctly folded and misfolded G1M9-glycoproteins [10]. We considered that such a mixture could be a model for G1M9glycoproteins that escape from the ER-glycoprotein quality control system to the Golgi apparatus. Therefore, we employed it to evaluate the endo- $\alpha$-mannosidase substrate preference in a competitive digestion assay.

\subsection{Preparation of M9-glycosyl-polypeptides}

Our assay used crambin [11] as a small model protein, which can be conveniently modified to generate correctly folded and misfolded glycoproteins in a controlled and reproducible manner, as 
described in details in a previous publication $[10,12]$. Herein we briefly summarize their syntheses, whereas the full details can be found in the original publication [10]. Natural crambin is a nonglycosylated protein consisting of 46 amino acid residues. We intentionally inserted a Man9-high mannose type oligosaccharyl asparagine (M9-Asn) (Figure 3b) at the position 17 estimating that this modification would not disturb the correct folding of crambin (1, Figure 3a). Native crambin also has three disulfide bonds (Cys 3-40, 4-32, and 16-26) [10, 11, 13]. In order to generate a set of misfolded species we attempted to disrupt the original disulfide bond patterns by replacing two of the six cysteines with serine (2, Figure 3a). Folding in vitro of the modified glycoprotein model successfully yielded three kinds of misfolded glycosyl crambins as shown in our previous publication [10].

M9-Glycosyl-crambin-polypeptide $\mathbf{1}$ and its mutant $\mathbf{2}$ were synthesized by solid phase peptide synthesis (SPPS) followed by native chemical ligation (NCL) [10]. The cysteine at the 16 position was used for the two segments coupling strategy by NCL. The N-terminal peptide- $\alpha$-thioester (TTCCPSIVARSNFNA- $\alpha$-thioester) and the C-terminal glycopeptide bearing a Man9-highmannose type oligosaccharide (CN(M9)LPGTPEALCATYTGCIIIPGATCPGDYAN) were synthesized by Boc-SPPS and Fmoc-SPPS, respectively. The Asn-M9-highmannose type oligosaccharide was isolated from egg yolk and then converted into Fmoc-Asn-(M9-highmannose type oligosaccharide)$\mathrm{OH})$. Its structure was confirmed by NMR analyses in comparison with a chemically synthesized sample [12]. NCL successfully produced M9-glycosyl-crambin polypeptide $\mathbf{1}$ in good yield. To achieve the glycosylated peptide mutant 2, Cys3 and Cys4 were both substituted with Ser during Boc-SPPS and then used for NCL [10].

Using the previously established conditions [10], we examined the simultaneous folding of M9glycosyl-crambin polypeptide $\mathbf{1}$ and its mutant $\mathbf{2}$ in the presence of UGGT and UDP-glucose, mimicking the ER folding process. M9-glycosyl-crambin polypeptide 1 only produced the correctly folded M9-glycosyl-native-crambin 3, with three disulfide bonds, whereas the mutated M9glycosyl-crambin polypeptide $\mathbf{2}$ produced the predicted three species $\mathbf{4}, \mathbf{5}$ and $\mathbf{6}$ (Figure 3c). As 
previously reported, we identified by ESI-MS that products $\mathbf{4 , 5}$, and $\mathbf{6}$ have two disulfide bonds and a comparison of their CD profiles with that of M9-glycosyl-native-crambin 3 indicated that they were indeed in the misfolded form [10].

a)

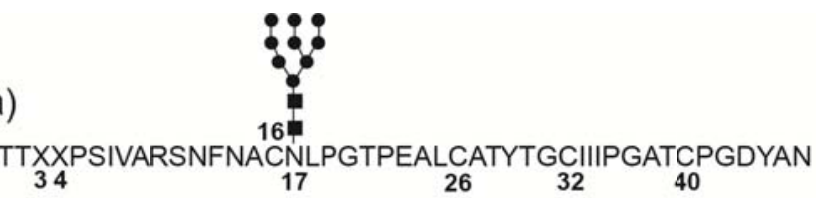

1: $X=$ Cys

2: $\mathrm{X}=\mathrm{Ser}$

c)

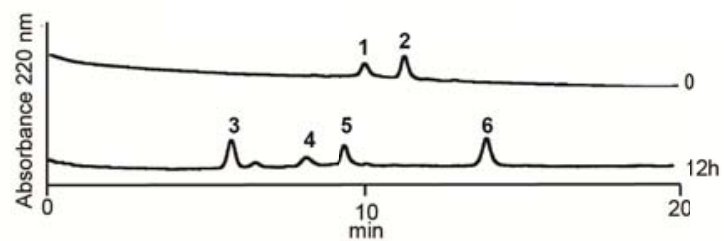

b)

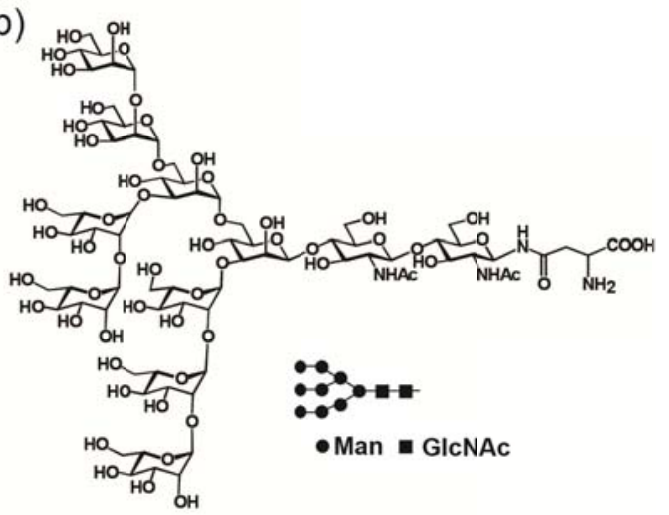

Figure 3. a) Amino acid sequence of crambin with the high mannose oligosaccharide inserted at position 17. Native (1) and mutated (2) sequences of glycosyl crambin; b) High-mannose structure; c) UV-HPLC profiles for the monitoring of simultaneous folding of native (1) and mutated (2) glycosyl crambin. Peak 3 corresponds to the correctly folded glycosyl crambin and 4, 5 and $\mathbf{6}$ are the misfolded glycosyl crambins.

\subsection{Simultaneous preparation of G1M9-native and G1M9-misfolded glycoproteins by UGGT}

Further analysis of the folding products 3, 4, 5 and 6 using ESI-MS showed that all products were partially mono-glucosylated. The relative amount of glucosylation of each glycosyl-crambin was subsequently determined by comparing the ESI-MS peak intensity of individual spectra with those of the parent non-glucosylated species, Figure 4a: at 0 min the quantity of G1M9 oligosaccharide. Although glycosyl crambin 3 was correctly folded, we have already confirmed that UGGT transfers a glucose residue on the folding intermediates during its folding processes [10]. The glucosylation of the correctly folded and misfolded glycosyl-crambins was between 25 and 30 $\%$ (by comparison with the non glucosylated one) and these substrates were used for the enzymatic assay without further purification. 


\subsection{Endo- $\alpha$-mannosidase assays for M9-glycosyl-glycoproteins}

The assay was initiated by the addition of the endo- $\alpha$-mannosidase to the mixture of glycoproteins 3, 4, 5 and $\mathbf{6}$. The enzymatic activity of endo- $\alpha$-mannosidase was evaluated by LC/MS analysis, comparing the G1M9/M8 ratio over time. The endo- $\alpha$-mannosidase reaction was followed for 1 hour, during which time the relative percentage of G1M9 and M8 decreased and increased, respectively (Figure $4 \mathrm{a}$ and $4 \mathrm{~b}$ ).
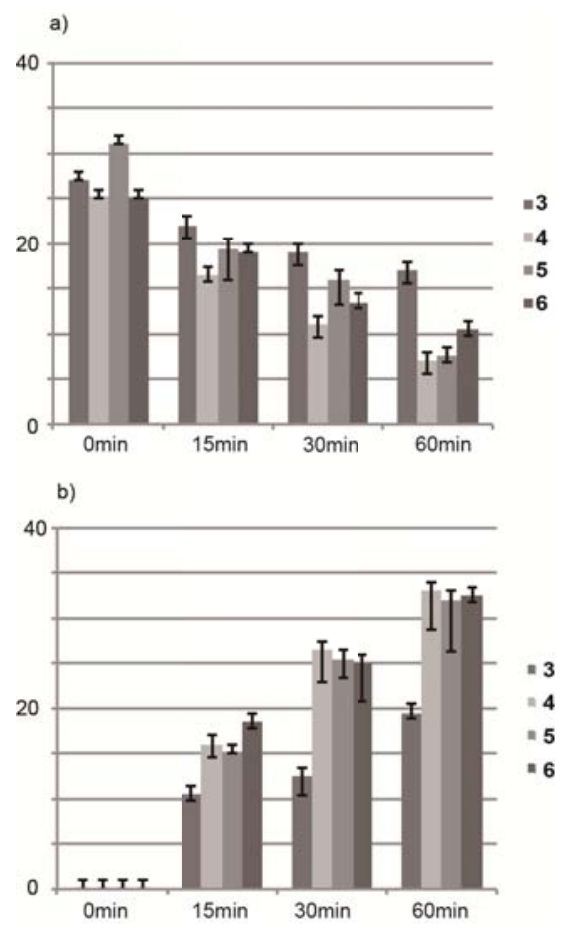

Figure 4. a) Decreasing of the percentage of G1M9 in the time course for the four species of glycosyl-crambins 3, 4, 5 and 6 ; b) increasing of the percentage of M8 in the time course for the four species of glycosyl-crambins 3, 4, 5 and $\mathbf{6}$. The longitudinal axis means relative percentage.

Figure $5 \mathrm{a}$ and $5 \mathrm{~b}$ show the ESI-MS spectra for the folded native sequence of glycosyl crambin 3 and for the folded mutated sequence $\mathbf{6}$, respectively. In both cases, the M9 peak corresponds to the molecular weight signal of the folded M9-glycoprotein, while the G1M9 peak corresponds to the molecular weight signal of the mono-glucosylated glycosyl crambin. During the endo- $\alpha$-mannosidase reaction, a new peak (marked M8) can be seen after 15 minutes (Figure 5a and 
5b). This corresponds to the molecular weight signal for the product of endo- $\alpha$-mannosidase activity, which cleaves the terminal Glc- $\alpha-1-3-$ Man disaccharide unit from the A-arm of the glycoprotein oligosaccharide (Figure 2).

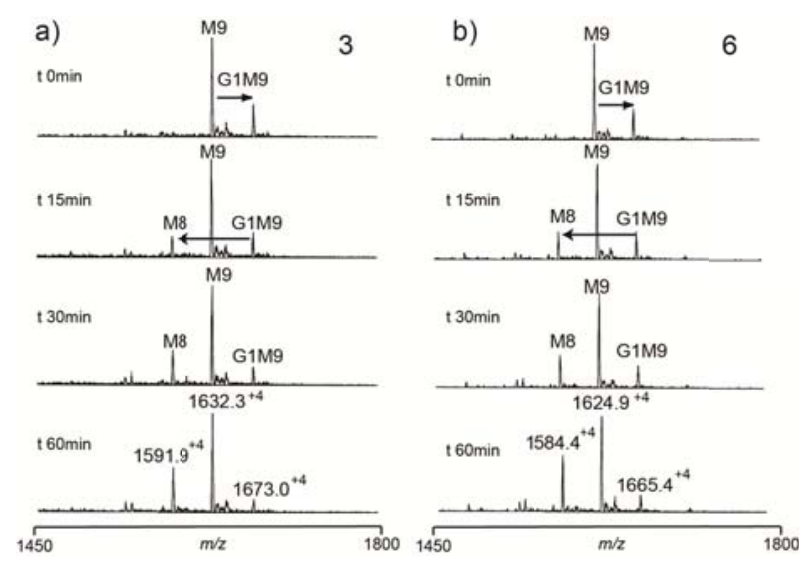

Figure 5. ESI-MS spectra for a) native sequence glycosyl crambin 3 and b) mutated sequence glycosyl crambin $\mathbf{6}$.

The results summarized in Figure 4 and Figure 5 clearly show that endo- $\alpha$-mannosidase hydrolyzes the Glc- $\alpha-1-3-$ Man unit from G1M9-high mannose type oligosaccharides, regardless of the folding state of the glycosyl crambin in which they are incorporated. The assay mixture included similar quantities of correctly folded glycosyl-crambin $\mathbf{3}$ and misfolded glycosyl-crambin 4, 5 and 6 (Figure 2), and endo- $\alpha$-mannosidase did not show a clear substrate preference toward correctly folded and three types of misfolded species.

\section{Discussion}

The alternative de-glucosylation route performed by Golgi endo- $\alpha$-mannosidase discovered by Spiro et al. [5,6] lead to the hypothesis that this enzyme could act as a back-up quality control system for the maturation of $N$-glycoproteins that elude UGGT/GII quality control in the ER [8].

Current dogma suggests that only correctly folded glycoproteins can be transported to the Golgi after the removal of the glucose tag in the ER, while all misfolded species are degraded by 
the ERAD system [1]. It was therefore intriguing for us to investigate further the role of the endo- $\alpha$ mannosidase, and in particular its target glycoproteins.

The substrate specificity of endo- $\alpha$-mannosidase has been previously examined by use of a heterogeneous mixture of glycoproteins; therefore, we thought that a more suitable analysis employing only homogeneous substrates would be valuable to confirm the obtained results. By folding the native and mutated M9-glycosyl-crambin in the presence of UGGT, it was possible to obtain the set of substrates required for our investigation. The preparation of individual isolated homogeneous G1M9-native- and misfolded-crambin is challenging due to the limited amount of glycoprotein substrates available and the difficulty in the HPLC separation between the monoglucosylated (G1M9-glycoprotein) and the residual non glucosylated (M9-glycoprotein) species. Nevertheless, our simple assay produced a mixture of G1M9-native-crambin and G1M9-misfoldedcrambin including their M9-forms and clearly showed that endo- $\alpha$-mannosidase did not discriminate between the different proteins. Although we started from equal quantities of unfolded M9-glycopeptides $\mathbf{1}$ and 2, the exact amount each final folded species 3, 4, 5 and $\mathbf{6}$ was not evaluated in this assay, therefore the results should be considered as a qualitative indication of the endo- $\alpha$-mannosidase substrate preference. However, endo- $\alpha$-mannosidase cleaved Glc- $\alpha-1-3-$ Man unit from G1M9-misfolded crambins 4, 5 and $\mathbf{6}$, which employed three types of protein structure due to different disulfide bond patterns. These data clearly indicates that endo- $\alpha$-mannosidase has a broad substrate preference.

This broad substrate preference of endo- $\alpha$-mannosidase is not surprising, in fact, comparing its structure with that of UGGT, it is clear that the two have a substantial difference in the molecular capacity, suggesting that a misfolded protein-species recognition site could be missing in the endo$\alpha$-mannosidase [7]. UGGT, which can recognize either misfolded or hydrophobic patch of proteins, has a molecular weight of $150 \mathrm{KDa}[1]$ versus the $56 \mathrm{KDa}$ [7] of the endo- $\alpha$-mannosidase.

With our experimental design, we pursued two goals. First was to investigate the substrate preference of endo- $\alpha$-mannosidase toward monoglucosylated correctly folded and misfolded 
glycoproteins using a simple and efficient assay system. Second, we wanted to show the versatility of our correctly folded/misfolded crambin assay, having previously demonstrated its efficiency in evaluating the UGGT activity toward glycoprotein folding intermediates [10]. Here we were able to expand the assay by simply adding the endo- $\alpha$-mannosidase to the mixture and evaluate its substrate preference. The assay could be performed without redox conditions or guanidine, usually unsuitable for the stability of enzymes

We would like to gradually increase the enzyme components in the correctly folded/misfolded crambin assay system and build a unique in vitro glycoprotein quality control assay system by adding, simultaneously or in successive steps, several enzymes, such as GI, GII, chaperones, Sep15 and thereof. Such a system, with its simplicity in both synthesis and handling, would be invaluable for deciphering the cascade of reactions that take place in the ER quality control system.

\section{Conclusion}

Folding the native and the mutated glycosyl crambin in the presence of UGGT yielded the desired mono-glucosylated correctly folded and misfolded species to be used as substrates for the Golgi endo- $\alpha$-mannosidase. According to our experimental results, the Golgi endo- $\alpha$-mannosidease does not have any preference towards correctly folded or misfolded substrates.

\section{Experimental}

\subsection{Preparation of human endo- $\alpha$-mannosidase in $E$. coli.}

A cDNA encoding 27-462 aa of human endo- $\alpha$-mannosidase was inserted into pCold I expression vector (Takara Bio., Otsu, Japan), which is constructed with the attachment of $N$-terminal His -tag. . Expression of the recombinant protein in E. coli BL21 cells having pGro7 chaperone plasmid (Takara Bio) and its purification using Ni-NTA agarose (QIAGEN) were performed according to the manufacturer's instructions. The yield of the protein was $3.9 \mathrm{mg}$ per $1 \mathrm{~L}$ culture.

5.2. Preparation of a mixture of mono-glucosylated crambins ( 1 and 2 ) and Endo-amannosidase assay 
A simultaneous folding of glycosyl-crambin polypeptide $\mathbf{1}$ and $\mathbf{2}$ in the presence of UGGT and UDP-glucose followed by endo- $\alpha$-mannosidase assays were performed in one-pot. Glycosylcrambin polypeptides $\mathbf{1}$ and $\mathbf{2}$ were folded in the presence of UGGT and UDP-glucose in a solution (50 mM Tris-HCl, pH 7.5, air bubbled for $30 \mathrm{sec}$ ) including $\mathrm{CaCl}_{2}(5 \mathrm{mM})$ and UDP-Glc $(0.5 \mathrm{mM})$, UGGT (human $4.5 \mathrm{ng} / \mu \mathrm{L}$ ) [10] and the mixture was incubated at $37{ }^{\circ} \mathrm{C}$. The concentration of glycosyl-crambin polypeptide 1 and 2 was adjusted at $15 \mu \mathrm{M}$. This condition led to start folding of glycosyl-crambins 1 and 2, and simultaneous glucosylation by UGGT [10]. The folding progress was monitored by LC/MS (Proteonavi $\Phi 2.0 \times 150 \mathrm{~mm}, 0.1 \%$ TFA : $0.1 \%$ TFA in 90\% MeCN $=75$ : 25 to $50: 50$ over $20 \mathrm{~min}$ at $0.2 \mathrm{~mL} / \mathrm{min}$ ). When the folding was complete, endo- $\alpha$-mannosidase (human $0.4 \mu \mathrm{g} / \mu \mathrm{L}$ ) was added in the reaction vial. At the specific time point, an aliquot of the enzymatic reaction mixture was taken, and diluted with the same volume of $0.1 \mathrm{M} \mathrm{HCl}$ to quench the enzymatic reaction. The samples were directly analyzed by LC/MS (Proteonavi $\Phi 2.0 \times 150 \mathrm{~mm}$, $0.1 \%$ TFA : $0.1 \%$ TFA in $90 \% \mathrm{MeCN}=75: 25$ to $50: 50$ over $20 \mathrm{~min}$ at $0.2 \mathrm{~mL} / \mathrm{min}$ ). Because all the G1M9, M9 and M8 glycoprotein forms of each specie eluted at the same retention time, the endo- $\alpha$-mannosidase activity was estimated by comparison between the mass peak intensity of each species present in the reaction mixture assuming that all the different glycoproteins have the same ionization properties. The sum of all the three species, M9, G1M9, M8 was considered to be the 100

$\%$, the relative amount of G1M9 and M8, this last one being 0 at $\mathrm{t}=0$, species was calculated accordingly to this assumption.

\section{Acknowledgment}

This work was supported by a Grant-in- Aid for the Exploratory Research for Advanced Technology (ERATO) from the Japan Science and Technology Agency (JST).

\section{References}

[1] A. Helenius, M. Aebi, Annu Rev Biochem 2004, 73, 1019-1049.

[2] R. Kornfeld and S. Kornfeld, Annu Rev Biochem 1985, 54, 631-664.

[3] C. D. Alessio, J. J. Caramelo, and A. J. Parodi, Semin. Cell Dev. Biol. 2010, 21, 491-499.

[4] R. G. Spiro, J Biol Chem 2000, 275, 35657-35660.

[5] W. A. Lubas and R. G. Spiro, J Biol Chem 1988, 263, 3990-3998.

[6] W. A. Lubas and R. G. Spiro, J Biol Chem 1987, 262, 3775-3781.

[7] A. J. Thompson, R. J. Williams, Z. Hakki, D. S. Alonzi, T. Wennekes, T. M. Gloster, and K. Songsrirote, J. E. Thomas-Oates, T. M. Wrodnigg, J. Spreitz, et al., Proc Nat Acad Sci USA, 2012, 109, 781-786.

[8] J. Roth, M. Ziak, and C. Zuber, Biochimie 2003, 85, 287-294.

[9] T. Torossi, J. Y. Fan, K. Sauter-Etter, J. Roth, and M. Ziak, Cell. Mol. Life Sci. 2006, 63, 1923-1932.

[10] S. Dedola, M. Izumi, Y. Makimura, A. Seko, A. Kanamori, M. Sakono, Y. Ito, and Y. Kajihara, Angew. Chem. In.t Ed. 2014, 53, 2883-2887. 
[11] C. H. VanEtten, H. C. Nielsen, and J. E. Peters, Phytochemistry 1965, 4, 467-473.

[12] Y. Makimura, T. Kiuchi, M. Izumi, S. Dedola, and Y. Ito, Y. Kajihara, Carbohydr Res 2012, 364, 41-48.

[13] Y. Kajihara, Y. Tanabe, S. Sasaoka, and R. Okamoto, Chem. Eur. J. 2012, 18, 5944-5953. 
